\title{
Always look on the bright side of the fluorophore
}

In super-resolution microscopy, dynamically adapting the illumination of the sample to the imaged features boosts contrast and resolution, as Stefan Hell and colleagues report in the Proceedings of the National Academy of Sciences, USA.

Super-resolution microscopy is a powerful tool for the investigation of biological samples, resolving details that are much closer together than the diffraction limit ( $200 \mathrm{~nm}$ for visible light) and enabling analysis of whole cells. The features of the sample are tagged with fluorophore molecules that are excited by a laser beam and then detected. In conventional microscopy, all fluorophores separated by less than the diffraction limit are excited and imaged together. A popular super-resolution microscopy technique is stimulated emission depletion (STED) microscopy, in which a STED beam shaped like a doughnut is superimposed on the excitation beam, switching all molecules off except for the one molecule (or a few molecules) at the centre of the doughnut. This enables the recording of the signal from a single molecule at a time; by scanning the STED beam across the sample, all molecules are eventually imaged.

The limitation of this approach is that the light intensity in the region where the excitation spot and the doughnut crest overlap can be much higher than that required to switch the fluorophores off. The extra intensity causes photobleaching; that is, the molecules are chemically damaged and become unable to fluoresce. Thus, the theoretical resolution of STED microscopy has not yet been reached.

The new imaging mode, dubbed dynamic intensity minimum
(DyMIN), reduces sample exposure, using only the amount of light that is needed. For two fluorophores that are closer together than the diffraction limit, the process starts with a diffraction-limited probing step, in which the excitation beam (without STED light) is moved towards the first molecule until this starts to emit. At this point, the STED beam is switched on but only at the minimum intensity needed to switch off the molecule. In steps, the beam is brought closer to the molecule while the STED intensity is increased to keep it in the off state; resolution increases at each step, until the molecule is at the centre of the doughnut and emits, while its companion is still off. The fluorophores receive a much lower amount of STED light than in conventional scanning, in which the STED intensity is set to full power all the time. The lower exposure of the sample reduces bleaching; thus, the images are brighter and the resolution higher.

To compare DyMIN with other STED concepts, the authors performed simulations to estimate the average amount of STED light received by molecules. The systems that were considered included random distributions of points, crossing lines (as in cytoskeletal filaments) and circles (as in virus particles or vesicles). Already with three DyMIN steps there was a remarkable reduction in STED dose; more steps further reduced the total illumination.
Hell and colleagues used DyMIN to image biological samples, such as the distal axon in neurons and synapses, and observed a 10-fold increase in brightness and a 20-fold (or even 100-fold for sparser samples) lowering of the light dose. They could image structures with a lateral separation as small as $30 \mathrm{~nm}$. DyMIN can also be used for multichannel recording, in which dyes emitting at different wavelengths are used.

There is margin to further improve this technique. For example, it would be good to establish early whether a fluorophore is present or not to be able to skip areas without fluorophores; this could be done by starting from a low-resolution image of the fluorescent structures.

Giulia Pacchioni

ORIGINAL ARTICLE Heine, J. et al. Adaptiveillumination STED nanoscopy. Proc. Natl. Acad. Sci. USA http://dx.doi.org/10.1073/pnas.1708304114 (2017)

FURTHER READING Sahl, S. J., Hell, S. W. \& Jakobs, S. Fluorescence nanoscopy in cell biology. Nat. Rev. Mol. Cell Biol.http://dx.doi.org/10.1038/ nrm.2017.71 (2017) 